Voix et Images

\title{
Un événement politique et sa représentation cinématographique
}

\section{Gilles Thérien}

Volume 1, numéro 1, septembre 1975

Hubert Aquin

URI : https://id.erudit.org/iderudit/013994ar

DOI : https://doi.org/10.7202/013994ar

Aller au sommaire du numéro

Éditeur(s)

Les Presses de l'Université du Québec

ISSN

0318-9201 (imprimé)

1705-933X (numérique)

Découvrir la revue

Citer cet article

Thérien, G. (1975). Un événement politique et sa représentation cinématographique. Voix et Images, 1(1), 135-142.

https://doi.org/10.7202/013994ar d'utilisation que vous pouvez consulter en ligne.

https://apropos.erudit.org/fr/usagers/politique-dutilisation/ 


\section{Un événement politique et sa représentation cinématographique}

L'événement dont il sera ici question est ce qu'il est convenu d'appeler la crise d'octobre 70. Les films sont Bingo du réalisateur Jean-Claude Lord et les Ordres du réalisateur Michel Brault. Le sujet est évidemment piégé. On s'est déjà beaucoup exprimé, et avec passion, tant sur la crise elle-même que sur les deux films. Mon propos ne sera donc pas d'ajouter une nouvelle "version" à la liste déjà longue et aux positions irréconciliables, mais de faire l'examen du fait et de sa représentation à l'intérieur d'un cadre sémiologique. Il s'agit de scruter les films et d'analyser le type de rapport qu'ils entretiennent avec les faits.

\section{QUESTION DE MÉTHODE}

Des critiques de type herméneutique ont été faites à propos des deux films. La part subjective a parfois été si grande que le critique parlait autant de lui que du film. Il faut se garder d'une pareille confusion.

Le premier objectif est de délimiter une chaîne d'événements qui rendent compte de la crise d'octobre en ce qui concerne son déroulement, le début étant constitué par l'enlèvement de James Richard Cross et la fin, par sa libération. Nous aurons alors ce que j'appelle l'axe de l'événement constitué d'un certain nombre de récits qui n'ont pour but que de rendre cet événement accessible. II faudra donc éliminer de cet axe tout récit à caractère subjectif - et idéologique - pour ne conserver que ce qui constitue la mémoire même de cette tranche de notre histoire.

Pour les commodités de l'analyse, posons cet axe horizontal, identifiant ainsi une sorte de déroulement, de "bande" d'événements. Ce déroulement n'a ni caractère évolutif, ni caractère causal. II se présente comme chronologie et l'ordre de ce qui y est dit n'est qu'un ordre d'apparition dans le temps chronologique.

Les films, eux, sont placés sur un autre axe, perpendiculaire au premier, et ne font pas partie d'une chronologie d'événements mais plutôt d'une représentation, au sens très large que ce mot peut avoir. La littérature, le théâtre, une exposition de photos, peuvent aussi représenter ces événements. L'univers de la représentation est anhistorique et possède ses 
lois propres, dictées par le médium utilisé, l'auteur ou le public (la mode). La représentation n'est en aucune sorte reproduction des événements. Elle implique donc chaque fois transformation et, par voie de conséquence, manipulation. Ce dernier terme n'indique que l'opération consistant à refaire un ordre, à laisser de côté ou à intégrer des événements.

L'axe de représentation concerne le public en tant que spectateur. Les résultats escomptés sur l'axe des événements tiennent surtout à l'espoir qu'ont les auteurs d'avoir une influence sur le déroulement de la vie. La plupart du temps, cet espoir est déçu et les spectateurs ne modifient au mieux que leur façon de voir rétrospectivement un fait, vécu ou non.

Ce que nous examinerons ici, c'est donc uniquement le point de rencontre des deux axes, c'est-à-dire là où l'événement devient représentation, spectacle. Nous tenterons d'indiquer le type de transformation et la façon dont il se présente dans l'univers filmique, puis l'idéologie qui se dégage du système de signes employé.

\section{L'AXE DES ÉVÉNEMENTS}

Les événements d'octobre s'étendent en fait du 5 octobre au 3 décembre, c'est-à-dire de l'enlèvement de James Richard Cross à sa libération. II y a bien sûr eu un "avant " du FLQ et un "après " de la crise (caractérisé par une sorte d'apathie qui subsiste toujours). Toutefois, par rapport aux deux films analysés, nous utiliserons surtout la période stricte de la crise. En voici le résumé, au tableau 1.

\section{TABLEAU 1}

\section{OCTOBRE}

le 5

(a) Enlèvement de Cross

(b) Conditions du FLQ

(c) Conférence de Choquette

(d) Entente OttawaQuébec

(e) Arrestations

(f)

\section{Le 11}

(a)

(b)

(c) Offre de négociation
107

le 8

Diffusion du manifeste du FLQ

Ouverture de la session parlementaire

Convoi militaire de Valcartier à Sainte-Thérèse

1017 le 16 le 25 Mort de Pierre Laporte

le 10

Enlèvement de Pierre Laporte

Refus des conditions posées par le FLQ 
(d)

(e)

(f)
Lois des mesures

de guerre

Arrivée de

l'armée -

arrestations

Lettre de Drapeau
Réélection massive de Drapeau

\section{DÉCEMBRE}

le 3

(a) Libération de Cross

(b) Départ des membres du

FLQ vers

Cuba

Notons une structure générale de (5a), l'enlèvement de Cross, à (3a), sa libération. Ce seul acte aurait suffi amplement à engendrer toutes les réactions relevées dans le tableau. II est presqu'inévitable de considérer l'enlèvement et la mort de Pierre Laporte (10a à 17a) comme une parenthèse qui a principalement servi à augmenter la tension, les pressions. C'est la mort de Laporte qui va mettre un terme à ce qu'une grande partie de la population considérait comme un spectacle inattendu.

Les deux victimes représentent chacune les pôles de notre société. Le conquérant, l'Anglais, le conquis, le Québécois. Comme on l'a relevé à l'époque, c'est encore le Québécois qui a payé. Trois gouvernements sont touchés: le fédéral, responsable de la protection des diplomates, le provincial, solidaire du ministre Laporte, et le municipal qui prépare pour le 25 octobre la réélection de Jean Drapeau contre une opposition populaire, le FRAP.

Le refus d'Ottawa face à la demande des felquistes aurait dû entraîner la mort de Cross, c'est pourtant Laporte qui meurt à la suite du refus du gouvernement du Québec.

La police a mis exactement trente jours à trouver Cross, ce qui est beaucoup et elle n'a pu localiser Pierre Laporte. Les arrestations, les perquisitions avec ou sans saisie ont semblé être parfois reliées à des options politiques, parfois au hasard, à l'époque nommé plus justement délation. Toutes les pistes ont été suivies... même les fausses, minutieusement.

C'est la représentation de ces événements que nous allons tenter de retrouver dans les deux films.

\section{L'AXE DE LA REPRÉSENTATION}

Le film, quel qu'il soit, recrée un environnement, un espace qui se donne pour la réalité, la réalité du vécu mais aussi de ce qui est vécu. En ce sens, le film est représentation. La nature même du médium s'y présente 
comme une représentation visuelle dont les valeurs narratives appartiennent à l'écriture cinématographique qui sait insister sur tel ou tel trait.

La sémiologie du cinéma se doit d'examiner l'image à trois niveaux: l'iconique, l'indiciel et le symbolique. L'iconique rend compte de l'image, supportée par la pellicule, avec toutes ses caractéristiques descriptives et visuelles. Telle couleur, tel type de pellicule, tel cadrage, tel éclairage sont autant de facteurs qui viennent désigner la nature même de l'image filmique. L'indiciel analyse le rapport qui s'établit entre la technique et la narration: les choses sont montrées, de près, de loin, avec une certaine insistance, sous un angle particulier. Enfin le symbolique renvoie à l'univers connotatif des films d'un auteur, de l'influence d'un auteur sur un autre ou d'une culture. Ces caractères de l'image seront utilisés ici. Ils prendront appui sur le kinogramme, c'est-à-dire la portion de pellicule comprise entre deux coupures. C'est ainsi que je nomme l'unité d'analyse d'un film sans me soucier de savoir si c'est l'unité de base du code, cette question étant prématurée dans l'état actuel de la sémiologie du cinéma.

II ne sera pas possible de faire ici un compte rendu kinogramme par kinogramme des films, et d'ailleurs cela n'est pas essentiel. Que l'analyse parte du plus petit pour arriver à des grandeurs synthétiques ou l'inverse, la seule chose importante est de s'en tenir au film. Enfin si nous évitons les concepts de syntagme ou de séquence, c'est que nous ne croyons pas qu'ils puissent éclairer la compréhension d'un film. Une série de kinogrammes peut être reliée à une autre et cela suffit pour l'analyse.

\section{BINGO}

La narration de Bingo de Jean-Claude Lord se situe à trois niveaux: l'histoire d'une famille, l'histoire sociale d'une grève et de ses conséquences, I'histoire d'une élection. En voici un résumé, au tableau 2, selon ces trois niveaux: à chaque ensemble (a) (b) (c), correspond un macro-kinogramme, c'est-à-dire un épisode du film.

\section{TABLEAU 2}

(a)

(b)

(c) François-Geneviève Eugène-chômeur (premier début)

Présence du député Piquetage - incidents - mort de Léo

(deuxième début)

(a) Apparition de Pierre

Radicalisation des travailleurs - prise de cinq otages Problèmes familiaux d'Eugène

(a) Campagne électorale

(b)

(c) Implication de François

(a) Utilisation de la violence par les politiciens - scandales

(b) Explosion d'une bombe/trois morts

(c) Double complicité tacite François/Eugène, François/Geneviève 
(a) Avance du parti de droite

(b)

Fernand, berné, cherche à tuer Léger Fin des activités...

(c)

(a) ... de la cellule terroriste Pierre dénonce François
(c)

(a) Victoire de Léger Rencontre de Léger et de Pierre en

(b) Victoire policière

(c) Éva dénonce François - mort de François

(première fin) (deuxième fin)

(a) Niveau politique

(b) Niveau social

(c) Niveau familial

\section{A.}

Ce film, tourné en couleurs, avec une vedette internationale-de classe $B$, Alexandra Stewart, (d'origine québécoise, il va sans dire!) se donne toutes les allures de la fiction et qui plus est de la fiction hollywoodienne avec cascadeur patenté. Sur le plan iconique, le film est bien fait. La caméra est mobile, les images n'ont rien de narcissique; elles viennent s'insérer tour à tour dans la narration. Les comédiens sont bons, sauf Claude Michaud à qui l'exaltation syndicaliste ne convient pas trop. Dans Bingo, Duceppe excelle. Serait-il plus près de sa vérité ? Enfin François et Geneviève gardent la parité de leur anonymat.

Jean-Claude Lord sait raconter. II se préoccupe beaucoup du niveau indiciel. Il porte attention au spectateur et s'assure que ce dernier comprend et le suit. Il utilise bien les plans d'ensemble et les plans moyens et se garde d'une utilisation excessive des gros plans. Les indices sont donnés de sorte qu'un doute sur la véritable trame narrative puisse exister, c'est le cas de Pierre dont les premières apparitions furtives sont remarquées et demeurent inexplicables.

Quant au niveau symbolique, il est peu important si on cherche dans les détails. Toutefois l'ensemble du film se veut calqué sur État de siège et $Z$ de Costa Gravas. C'est un choix.

B.

La comparaison entre cette représentation et les événements est très intructive. Des événements de 70 , Lord ne garde que l'enlèvement, l'élection, la libération des otages. Et encore là, ce ne sont que des structures: les otages ne sont plus des hommes politiques en poste mais des financiers (deux Anglais, deux Québécois et un Italien) qui, comme bien d'autres, ont des relations occultes avec le parti au pouvoir, l'élection n'est plus municipale mais provinciale. Le gouvernement fédéral est absent et l'élection préoccupe trop les partis pour qu'on songe à instaurer un pouvoir policier. La libération des otages est escamotée et laisse croire.qu'ils retourneront tous vaquer à leurs occupations habituelles. 
Sont ajoutés les actes de guérilla, les bombes en particulier, l'utilisation des photos compromettantes du ministre de la Justice et l'accident inévitable quand on pose des bombes, la mort.

Les bombes, les morts accidentelles font partie de l" «avant " de la crise d'octobre 70 . Les premières vagues felquistes se sont spécialisées dans les boittes aux lettres, les porches d'immeubles gouvernementaux et les statues-symboles de notre colonisation, Wolfe, la reine Victoria. Ceci, relié à des luttes syndicales, rappelle les événements des années 60 .

Lord fait aussi l'hypothèse que la violence profite aux partis de droite et qu'elle entraîne une démobilisation de la population. Éva, la spécialiste du bingo, espoir du pauvre, dénonce «sans le vouloir» son petit-fils qui a déjà été "donné» par Pierre, l'ex-agent syndical, devenu provocateur officiel. La dialectique de la crise de 70 est perdue. II ne reste qu'un constat d'échec, représenté par l'élection du candidat Léger, sosie d'Yvon Dupuis. La conclusion du film, c'est moins la brutalité de la mort de François et de Geneviève que la corruption politique signifiée par la rencontre de Léger et de Pierre quelque part en Amérique centrale, là où traditionnellement se terrent les fascistes étrangers.

\section{LES ORDRES}

Le film de Michel Brault a une structure fort simple, linéaire, fondée sur une pseudo-fiction - pseudo-documentaire en couleur et en noir et blanc qui se donne pour but de suivre certains résultats de la crise d'octobre par le truchement de témoignages personnalisés.

Résumons, au tableau 3, le propos de Brault, selon les deux niveaux, policier et socio-familial. Notons que les textes de Trudeau et de Drapeau sont des "hors-film ".

\section{TABLEAU 3}

Texte de P.E. Trudeau

(a)

Présentation pseudo-documentaire des témoignants

Texte de J. Drapeau

(a)

(b) Présence de l'interviewer en voix " off»

(a) Arrestations - perquisitions

(b) Présentation des autres témoignants

(a) Détention - interrogatoires

(b) La vie quotidienne du prisonnier

(a) Fausse exécution - passage à tabac

(b)

(a)

(b) Mme Boudreau, libérée, parle au ministre

premier interrogatoire

Incidents en prison

Mort du père de Boudreau

Visite de Boudreau à son père

(a) Libération sans explication 
(b) Réaction agressive (retour au noir et blanc)

(a)

(b) ...confidences (retour au début)
Reprise des...

Plan sur les toits de la ville

(a) Niveau policier

(b) Niveau socio-familial

II est à noter que les textes, de même que le plan final du film ne font pas partie du témoignage proprement dit mais introduisent subtilement l'interviewer invisible qui est Michel Brault et qui aurait dû, comme les autres comédiens, dire «je m'appelle Michel Brault mais dans le film, je suis... (Denis Héroux, par exemple!) ».

A.

Film au budget modeste, les Ordres invente des solutions de rechange sur le plan iconique. On y utilise à la fois la couleur et le noir et blanc. La réalité quotidienne est en noir et blanc, la prison en couleurs. Les images sont très bien faites mais leur agencement répond le plus souvent à des liens abstraits qui trouvent leur justification dans le commentaire. Sur le plan indiciel, le film est tout marqué des techniques de cinéma-vérité. Les gros plans abondent. Les actions sont détaillées, particulièrement en prison. L'ensemble des gestes accompagne le témoignage. Tout contribue à renforcer ce dernier. La proximité des visages, la chaleur que dégage l'image font du film un document qui «parle au cœur». Sur le plan symbolique, on reconnaît les traits de la caméra de Brault, attendrissement, cadrage recherché, douceur. Le film ne cherche pas à en copier un autre. II se présente pour lui-même.

B.

La linéarité des Ordres est loin de la relation des événements de la crise d'octobre. L'enlèvement de Cross, celui de Laporte de même que sa mort sont absents. II reste les lois des mesures de guerre, les arrestations, la détention. L'appareil politique de même que le judiciaire sont complètement disparus. Les témoignages ne sont pas ceux de felquistes mais de braves gens aux tendances vaguement socialisantes ou simplement anarchiques. L'aspect le plus inattendu est peut-être l'accent sur l'incarcération qui, au niveau des méthodes, tend à prouver qu'il n'y a pas de prisonniers politiques.

Michel Brault s'intéresse à une fraction de ceux qui ont été arrêtés en 70 et qui représentent une fraction de la majorité silencieuse, favorable au PQ. La stature politique des individus est faible.

L'aspect politique des événements se retrouve sous forme de texte dans les Ordres. C'est le texte de P. E. Trudeau, puis la lettre de J. Drapeau demandant l'intervention de l'armée et d'Ottawa. Le gouvernement provincial disparaît, ce qui confirme la thèse de René Lévesque en 70 sur l'inexistence d'un gouvernement responsable. Même le ministre de la Justice qu'on ne voit pas - ne semble pas capable de décider si Clermont Boudreau pourra aller voir son père décédé. 
Le film contient des incongruités graves. Que penser du syndicaliste Clermont Boudreau, joué par Jean Lapointe, auteur d'une chanson aux accents racistes pour le compte du parti libéral et qui chante aux dîners servis au bénéfice du même parti ? Le cadavre, fiction de son père, est en fait un vieux monsieur, petit-fils d'un des témoins de la révolution de 1837 dans un village des bords du Richelieu. Pourquoi occulter ce fait - fruit du hasard - et laisser la fiction l'affubler du. nom de Boudreau et le déplacer au Lac Saint-Jean? Mentionnons enfin que nombre de figurants tenaient des rôles analogues dans Bingo... surtout du côté policier!

\section{LES DEUX FILMS ET OCTOBRE 70}

Les deux films tronquent la réalité. Les deux font profession de fiction. Les deux prennent partie pour une fraction de la majorité silencieuse, la droite chez Lord (un constat désespéré?), le centre-gauche chez Brault (un espoir?). Les deux prétendent exprimer une vérité québécoise, proche du peuple.

La comparaison de l'axe de l'événement et de l'axe de la représentation confirme la manipulation, la transformation à des fins subjectives. Heureusement, le spectacle se suffit à lui-même et l'un et l'autre films se regardent sans bailler. Le sujet n'est pas épuisé. II manque encore un film plus près des événements, qui sache en démontrer les méandres. La représentation raconte des choses simples alors que l'événement n'a pas de vertu narrative. Il est un réseau de complexités entre organismes.

En somme, deux bons produits de consommation qu'il faut payer pour voir et qui illustrent les vertus du bingo et de l'erreur judiciaire.

Gilles Thérien 